\title{
FORMAÇÃO DE PROFESSORES DE LÍNGUA ESTRANGEIRA SOBRE OBJETOS DIGITAIS DE ENSINO E APRENDIZAGEM: UM MAPEAMENTO SISTEMÁTICO DA LITERATURA
}

\section{FOREIGN LANGUAGE TEACHERS' EDUCATION ON DIGITAL TEACHING AND LEARNING OBJECTS: A SYSTEMATIC LITERATURE MAPPING}

\author{
Francieli Motter Ludovico ${ }^{1}$, Patrícia Da Silva Campelo Costa Barcellos ${ }^{2}$
}

\begin{abstract}
RESUMO: Os processos de ensino e aprendizagem de Língua Estrangeira (LE) mediados pela Tecnologia de Comunicação Digital (TCD) podem e devem ir além da leitura na tela de um dispositivo digital, ou seja, esses recursos podem ser usados para encontrar outros caminhos. A TCD oferece diversas possibilidades, as quais permitem promover interação, colaboração e autonomia para que o estudante seja ativo e participe na construção do próprio conhecimento. Nesse sentido, os Objetos Digitais de Ensino e Aprendizagem (ODEA) configuram-se como possibilidades para mediar o ensino e a aprendizagem de LE. Para tanto, os professores precisam compreender o universo desses recursos. Nesse sentido, o presente Mapeamento Sistemático da Literatura ocupou-se de realizar um levantamento dos ODEA existentes para a formação de professores. Os resultados agregam discussões e contribuições para o delinear da formação de professores de LE sobre o uso e criação de ODEA para suas práticas.
\end{abstract}

PALAVRAS-CHAVE: ODEA; Ensino e Aprendizagem de Língua Estrangeira; Mediação; Tecnologia de Comunicação Digital.

ABSTRACT: The processes of teaching and learning a Foreign Language (FL) mediated by Digital Communication Technology (DCT) can and must go beyond reading on a digital device screen, in other words, these resources can be used to find other paths. DCT offers several possibilities, which allow promoting interaction, collaboration and autonomy so that the student is active and participates in the construction of his own knowledge. In this sense, the Digital Teaching and Learning Objects (DTLO) are configured as possibilities to mediate FL teaching and learning. Therefore, teachers need to understand these resources universe. In this sense, the present Systematic Literature Mapping carried out a survey of the existing DTLO for teacher education. The results aggregate discussions and contributions to outline FL teachers' education on the use and creation of DTLO for their practices.

KEYWORDS: DTLO; Foreign Language Teaching and Learning; Mediation; Digital Communication Technology.

\footnotetext{
${ }^{1}$ Doutoranda em Informática na Educação na Universidade Federal do Rio Grande do Sul

${ }^{2}$ Doutora em Informática na Educação e em Linguística Aplicada
} 


\section{Considerações Iniciais}

Já é de conhecimento popular que a Tecnologia de Comunicação Digital (TCD) encontra-se cada vez mais inserida em todas as atividades e contextos cotidianos. Logo, é hora de repensar o conceito de integrar tecnologia ao currículo e, em vez disso, procurar incorporar a tecnologia à pedagogia, para apoiar o processo de aprendizagem (EADY; LOCKER, 2013). Possibilitar a mudança das metodologias, não apenas no meio, pois conforme explica Lévy (1999, p. 172, grifos do autor), não se refere a "usar as tecnologias a qualquer custo, mas sim de acompanhar consciente e deliberadamente uma mudança de civilização".

Os recursos tecnológicos podem servir como instrumentos mediativos, os quais auxiliam na nossa ação. Ou seja, a aprendizagem "está estritamente vinculada ao processo de mediação, pelo qual o indivíduo atua no mundo através de dispositivos mediadores, os quais seriam instrumentos físicos e psicológicos" (COSTA BARCELLOS, 2014, p. 24).

Aspecto central da Teoria Sociocultural de Vygotsky (1991), a mediação "envolve sobremaneira ferramentas, além das relações interpessoais, de modo que o artefato tecnológico seja também um item mediador de conhecimento" (COSTA BARCELLOS, 2014, p. 28). Logo, a TCD pode auxiliar a aprendizagem, facilitando a interação social e a colaboração entre pares e é, por isso, considerada instrumento mediativo.

Uma das maneiras de possibilitar mediação por meio da TCD, é a elaboração e (re)uso dos Objetos Digitais de Ensino e Aprendizagem (ODEA). ODEA são recursos digitais, pequenos (uma foto, um vídeo, por exemplo) ou grandes (sites inteiros, que contém muitos ODEA pequenos, por exemplo), que possuem intenção de ensino, de criar situações de aprendizagem.

A criação e o (re)uso de ODEA nos processos de ensino e aprendizagem de LE possibilitam a descoberta de novas construções na língua, ao mesmo tempo em que cedem espaço para a dinamicidade, interatividade e colaboração, permitindo assim, a expressão da diversidade e a produção espontânea de tudo o que os participantes acreditam ser autêntico. Aspectos que podem mediar a aprendizagem da língua e, assim, 
auxiliar os estudantes a avançarem na sua Zona de Desenvolvimento Proximal (ZDP). ZDP, outro termo da Teoria Sociocultural que, conforme Vygotsky (1991) refere-se a distância entre o nível real de desenvolvimento, determinado pela solução independente de problemas, e o nível de desenvolvimento potencial, determinado pela solução de problemas sob orientação de adultos ou em colaboração com colegas mais capazes, experientes.

No entanto, construir ODEA é uma tarefa desafiadora (RAMOS; RAMOS; ASEGA, 2017), o que demanda multiletramentos por parte do professor, que conforme Rojo (2017, p.4),

(...) são as práticas de trato com os textos multimodais ou multissemióticos contemporâneos - majoritariamente digitais, mas também impressos -, que incluem procedimentos (como gestos para ler, por exemplo) e capacidades de leitura e produção que vão muito além da compreensão e produção de textos escritos, pois incorporam a leitura e (re)produção de imagens e fotos, diagramas, gráficos e infográficos, vídeos, áudio etc.

Assim, para criar e oferecer situações de aprendizagem de LE mediadas por tecnologias, os professores precisam de formação. Proporcionar esses momentos de reflexão e formação por meio dessas tecnologias pode contribuir na busca pela criação de referências para sua ação docente. Tendo em vista que as crenças dos educadores são arraigadas em suas vivências como aprendizes, assim se a referência é a transmissão e recepção de conhecimento, é muito provável que isso refletirá em suas futuras práticas (ZACHARIAS, 2016). Ludovico e Costa Barcellos (2019, p. 8) corroboram quando destacam que

\footnotetext{
para usar das TCD a favor da aprendizagem, essas devem ser centrais na formação docente (...) Logo, urge a necessidade da mudança nos cursos de formação de professores, momentos que ofereçam oportunidades para a reflexão e assim considerando a mudança de crenças e práticas, abram espaço para a invenção, para o crescimento humano da sociedade.
}

$\mathrm{Na}$ atuação enquanto mediador, ao professor cabe conhecer o contexto, os interesses e as histórias que os participantes trazem para o ambiente de aprendizagem. Assim, ao elaborar situações de ensino e aprendizagem mediadas por ODEA, o professor poderá oferecer práticas que propiciem multiletramentos para seus estudantes, o que conforme Rojo (2017, p. 8), é urgente, tendo em vista a necessidade de "formar 
pessoas, cidadãos e trabalhadores para o século em que estamos". Para tanto, formar professores para usar e construir ODEA para suas próprias práticas é uma ação necessária, pois conforme explica Rojo (2017, p. 6):

\begin{abstract}
o que nos interessa aqui, em termos de 'cenários futuros' para as escolas, é menos o espetaculoso da tecnologia, já existente mas ainda um tanto cara para nós, mas a metodologia de ensino-aprendizagem e os multiletramentos e gêneros que ela incorpora, típicos dos novos letramentos: um aluno que estudasse assim, certamente estaria mais preparado para a vida investigativa e colaborativa do mundo contemporâneo.
\end{abstract}

Dessa maneira, o presente artigo de revisão tem como objetivo mapear os Objetos Digitais de Ensino e Aprendizagem desenvolvidos para a formação de professores, a fim de verificar se algum deles é voltado para a autoria de ODEA e Língua Estrangeira e relatar resultados do estudo quanto à influência dos ODEA na formação de professores.

Até aqui, estabelecemos os princípios teóricos básicos que sustentam a pesquisa relatada neste trabalho. Nas próximas seções, apresentamos o percurso metodológico traçado pelas autoras e os resultados do mapeamento da literatura que examina a relação dos ODEAs e a formação de professores.

\title{
Percurso Metodológico
}

Este estudo caracteriza-se como um Mapeamento Sistemático da Literatura (MSL), um método de levantamento do estado da arte, utilizado quando o que é necessário não são respostas detalhadas para perguntas específicas, mas uma visão geral e ampla de uma área (MOHER, 2015). Moher (2015) considera que todos os tipos de revisão da literatura estão relacionados com a Revisão Sistemática da Literatura (RSL), a qual é o método mais consolidado. Dermeval, Coelho e Bittencourt (2017) explicam que as diferenças entre MSL e RSL encontram-se principalmente no foco da revisão. Uma RSL é conduzida a partir de uma questão de pesquisa do tipo causal, já o MSL é do tipo exploratório.

Dessa forma, dada a característica exploratória desta busca, optou-se por um MSL, considerando princípios do protocolo descrito por Kitchenham e Charters (2007), conforme representado na Figura 1: 
Figura 1 - Protocolo do Mapeamento Sistemático da Literatura

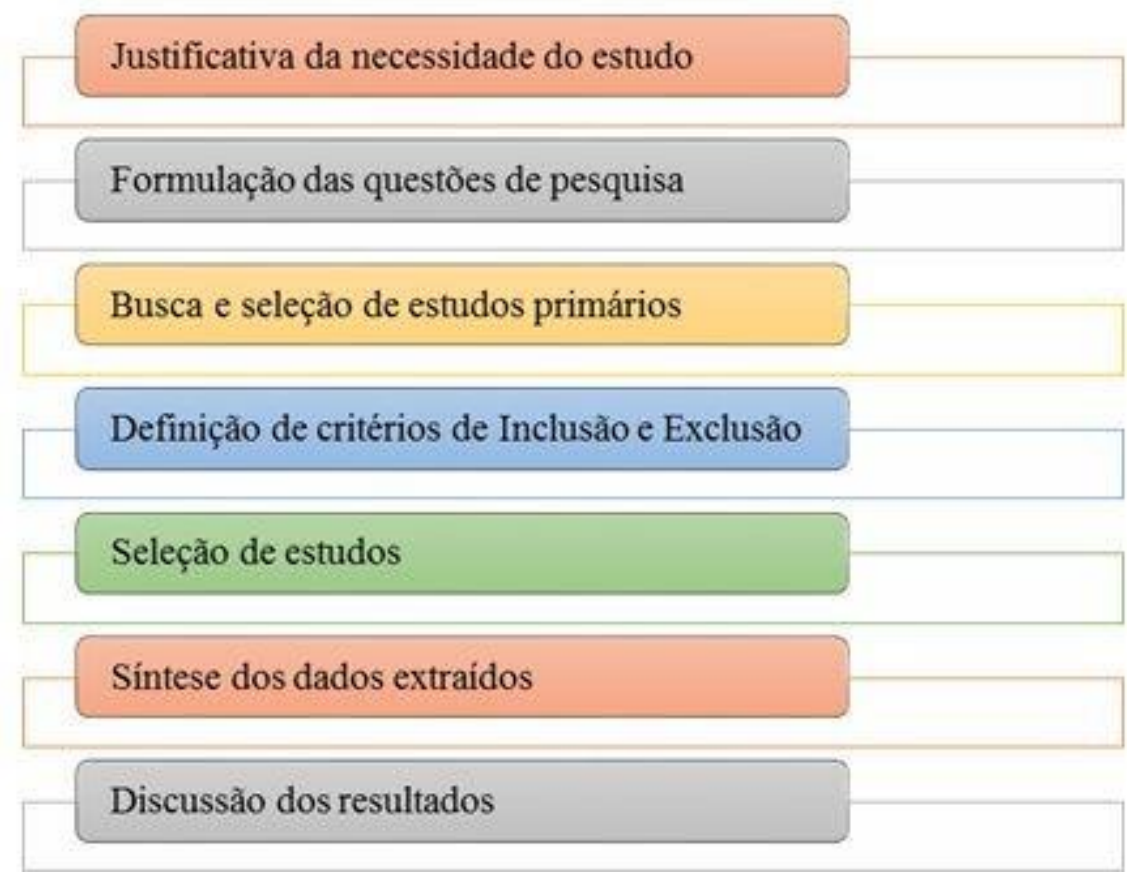

Fonte: Elaborada pelas autoras (baseada em Kitchenham e Charters, 2007).

Seguindo o protocolo, primeiramente, justifica-se a necessidade da realização desse levantamento, pois, embora existam RSL e MSL com a temática dos Objetos Digitais de Ensino e Aprendizagem, constatou-se que não há um estudo da literatura no que se refere ao uso desses recursos voltados à formação de professores.

Um MSL usualmente possui perguntas de pesquisa mais amplas que o direcionam e, em geral, acompanha várias perguntas de pesquisa (KITCHENHAM; CHARTERS, 2007). Assim, as questões norteadoras desse MSL são: Q1) Quais Objetos Digitais de Ensino e Aprendizagem (ODEA) desenvolvidos para a formação de professores existem? Q2) Entre esses objetos existe algum que é voltado para autoria de ODEA? Q3) Se sim, existe algum direcionado para Língua Estrangeira? Q4) Qual a influência dos ODEA na formação de professores?

A partir disso, as palavras-chave para o MSL foram definidas, compondo a string de busca: (“learning object?” OR “teaching object?” OR “digital resource?” OR “digital material?") AND (“teacher training” OR “teacher education” OR “pre-service teacher training” OR “pre-service teacher education”).

Destaco que termos relacionados à Língua Estrangeira não foram incluídos para não restringir a busca, a fim de encontrar maior número de contribuições. 
A busca foi realizada no portal de periódicos da $\mathrm{Capes}^{3}$, que dispõe de mais de 45 mil publicações, incluindo bases como SCOPUS, SpringerLink, IEEE, ACM Digital Library, Scielo, ScienceDirect, Web of Science, Wiley Online Library, entre outras.

Figura 2 - Critérios de inclusão e de exclusão

\begin{tabular}{|l|l|}
\hline \multicolumn{1}{|c|}{ Critérios de Inclusão } & \multicolumn{1}{c|}{ Critérios de Exclusão } \\
\hline (I1) Artigos completos publicados em & (E1) Artigos de revisão ou resumidos \\
periódicos & (E2) Artigos que apresentam pouca aderência \\
(I2) Artigos publicados entre 2008 e junho & ao contexto da pesquisa \\
de 2019 & (E3) Artigos duplicados ou redundantes \\
(I3) Artigos Revisados por pares & (E4) Artigo sem acesso gratuito ao seu \\
& conteúdo completo \\
\hline
\end{tabular}

Fonte: Elaborada pelas autoras.

Além dos termos de busca, foram inseridos filtros para definição do período considerado, de 2008 até junho de 2019, e para a seleção somente de artigos revisados por pares. Isso gerou um retorno inicial de 869 artigos. A partir da leitura do título, palavras-chave e resumo, os critérios de Inclusão e Exclusão (Figura 2) foram aplicados, resultando em 29 trabalhos para leitura completa. Para ser incluído o estudo deveria obedecer a todos os critérios de inclusão, mas para ser excluído, bastava que um dos critérios de exclusão fosse identificado.

Figura 3 - Processo de seleção dos artigos do Mapeamento Sistemático da Literatura

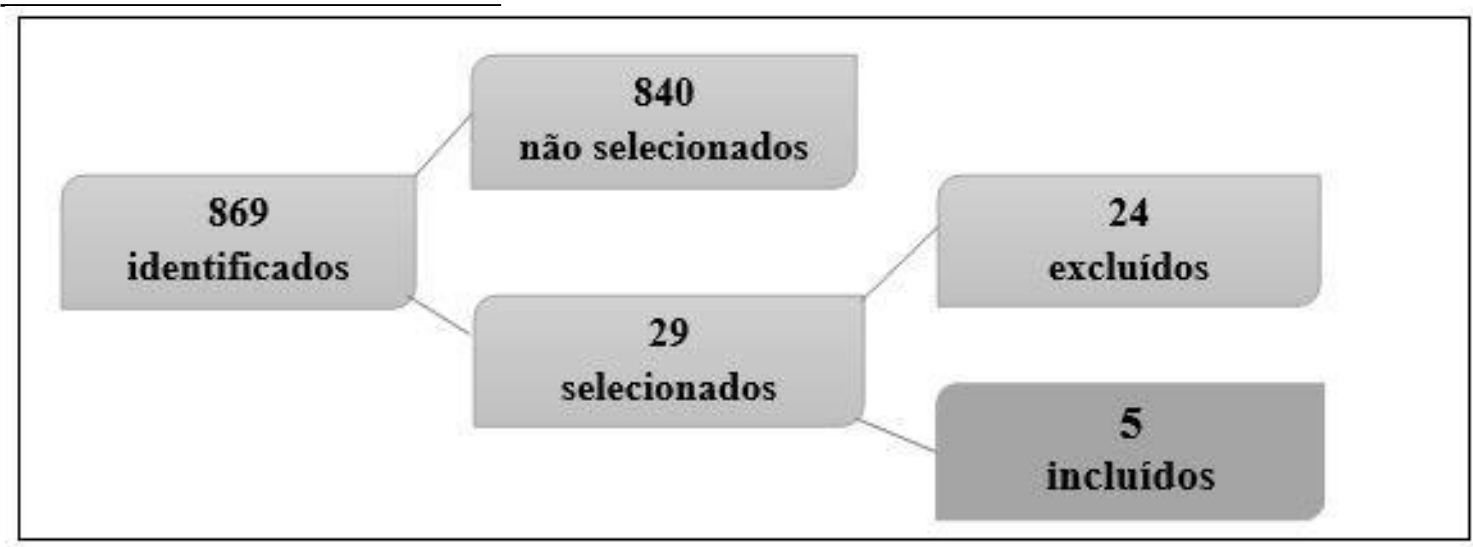

Fonte: Elaborada pelas autoras.

Após a fase de leitura, 24 artigos foram excluídos, restando cinco (05) estudos.

\footnotetext{
${ }^{3}$ Disponível em: https://www.periodicos.capes.gov.br/ Acesso em: jul./2020.
} 
O processo de seleção está representado na Figura 3. Dos cinco (05) trabalhos incluídos, três (03) são em inglês (BISOL; VALENTINI; BRAUN, 2015; ESTEBAN, 2018; PETRARCA, 2013), um (01) em italiano (LUPPI, 2013) e um (01) em espanhol (LÓPEZ; MARTÍNEZ; MONTOYA, 2014), os quais são apresentados na seção seguinte.

\section{Resultados e Discussões}

Para dar início a síntese dos resultados busca-se trazer pontos relevantes sobre cada um dos cinco (05) estudos selecionados por meio do Mapeamento Sistemático da Literatura. A apresentação dos artigos está em ordem de publicação, do mais antigo para o mais atual.

O trabalho de Petrarca (2013) explora como um pequeno grupo de professores de estágio acessou e interagiu com a Associate Teacher Learning Tool - ATLT (Ferramenta de Aprendizagem de Professores de Estágio). Essa é uma ferramenta de aprendizagem baseada na Web (Web-Based Learning Tool - WBLT) ${ }^{4}$ criada especificamente para professores de estágio, como uma abordagem alternativa para fornecer oportunidades de desenvolvimento profissional, fundamentada em uma estrutura teórica de prática reflexiva e aprendizagem construtivista. O ATLT final testado nessa pesquisa consistiu em 49 páginas baseadas em Flash, acessíveis através de um URL protegido por senha e organizado em torno de cinco temas principais que emergiram da literatura: 1) o papel de professor de estágio; 2) preparação; 3) planejamento; 4) feedback; e 5) práxis. As oportunidades de aprendizado ocorreram através de uma variedade de formatos de apresentação, documentação baseada em texto, gráficos, vídeos, atividades interativas e atividades reflexivas. No total, 17 professores de estágio participaram dessa pesquisa exploratória. Através da análise dos padrões de uso de professores associados e temas emergentes por meio do sistema de rastreamento interno da ATLT, os resultados desse trabalho revelaram que, embora os professores associados acessassem e interagissem com a ATLT de diversas maneiras, surgiram vários pontos em comum. A autora destaca que embora o trabalho não tenha explorado

\footnotetext{
${ }^{4}$ Petrarca (2013) usa o termo Web-Based Learning Tool - WBLT (ferramenta de aprendizagem baseada na $W e b)$ para fazer referência ao que aqui chamo de Objeto Digital de Ensino e Aprendizagem
} 
a aplicação real de qualquer aprendizado construído através do acesso e interação com o ATLT, os resultados são esperançosos com base nos padrões de uso e nos temas emergentes nas respostas reflexivas dos professores. Assim, o estudo conclui ser evidente que há potencial para uma forma de aprendizado baseada na Web ou desenvolvimento profissional, como o ATLT.

O estudo de Luppi (2013) tem como objetivo capacitar professores e educadores para o desenvolvimento sustentável por meio de ferramentas que podem ser utilizadas no e-learning. Para tanto, alguns Objetos de Aprendizagem foram desenvolvidos, para a modalidade semipresencial, usando diferentes ferramentas e estratégias para treinar as implicações educacionais do desenvolvimento sustentável. Cada OA possuía três núcleos de objetivos e conteúdo do tema: a) teórico; b) ético-filosófica e c) dimensão operacional prática. Do ponto de vista dos materiais e estímulos didáticos, optou-se por propor o mesmo conteúdo através de diferentes canais de comunicação (textos, imagens, diagramas e vídeos), constituindo hipertextos. Os OA contavam com o método estímulo-resposta representado por uma entrevista com uma pessoa privilegiada que oferecia uma introdução ao tópico tratado. Além disso, ofereciam exercícios para autoavaliação, por meio de perguntas de reflexão, construção de mapas conceituais e troca entre colegas. A autora justifica que, por tratar de um tema de natureza multidisciplinar, os OA permitiam aprimorar a construção de conhecimento por um aluno que era colocado em condições de escolher e traçar seu próprio caminho. Um total de 102 participantes respondeu ao questionário de satisfação em relação aos OA. Os dados do questionário revelaram resultados satisfatórios. A partir dos dados concluiu-se, também, que os OA são igualmente adaptáveis a diferentes contextos de ensino para fins, objetivos, configurações e metodologias de ensino. No entanto, ficou evidente que o uso dos OA depende, em grande parte, do contexto de ensino nos quais estão inseridos. Por fim, apesar de não ser o objetivo da autora, a partir dos questionários, foi possível perceber que os participantes aprenderam 'o valor dos Objetos de Aprendizagem', como uma ferramenta que promove a aprendizagem ativa com o objetivo de coconstruir conhecimento.

Em seu estudo, López, Martínez e Montoya (2014) buscam compreender até que ponto um Objeto de Aprendizagem (OA) elaborado para a formação docente orientado ao desenvolvimento de competências para usar Recursos Educacionais 
Abertos (REA) atende a um conjunto de critérios de qualidade pré-estabelecidos de acordo com o que caracteriza os $\mathrm{OA}$ abertos. $\mathrm{O}^{\mathrm{OA}^{5}}$ conta com os seguintes elementos: resumo, informações gerais (introdução, objetivo, agenda), diagnóstico e avaliação final, tópicos (atividade inicial, conteúdo, atividade final, recursos), referências e créditos. O conteúdo é apresentado em seis tópicos como uma pergunta, e o desenvolvimento do conteúdo de cada um foi escrito de forma independente. Ao final de cada tópico existe uma atividade final, exercício que permite validar o aprendizado, com feedback específico. A pesquisa de caráter qualitativo, do tipo exploratório, realizou validação com especialistas das áreas de conteúdo, pedagogia, design gráfico e tecnologia, que exploraram a relevância, escopo, objetivos de aprendizado, motivação, avaliação, usabilidade, acessibilidade, reutilização, sintaxe gráfica e semântica da OA. O questionário, respondido por 40 professores, explorou a relevância, escopo, objetivos de aprendizado, motivação, avaliação, usabilidade, acessibilidade, reutilização, sintaxe gráfica e a semântica da OA. Os resultados obtidos indicaram que o OA atendeu efetivamente às normas e especificações referentes aos aspectos pedagógicos e tecnológicos, porém houve outros aspectos, como concepções errôneas e problemas de revisão.

Bisol, Valentini e Braun (2015) apresentam uma avaliação do Objeto de Aprendizagem (OA) Incluir $^{6}$, criado para funcionar como uma ferramenta complementar para a formação de professores que visava promover a reflexão sobre inclusão e ressignificação da prática docente. A página inicial do objeto mostra uma imagem que representa um neurônio, por sua fácil associação com ideias de abertura e não linearidade: as conexões múltiplas e rizomáticas convidam imediatamente a um começo livre e não hierárquico e incentivam a mais navegação. Ao mesmo tempo, permite uma fácil agregação de novos módulos à medida que novos tópicos de interesse são desenvolvidos. Na tela do computador, a imagem se move e os efeitos visuais simulam sinapses ou trocas de energia. O OA está organizado em quatro módulos que integram recursos e materiais (vídeos, animações, imagens e textos) desenvolvidos por uma equipe. Cada módulo é independente dos outros em termos de argumento,

\footnotetext{
${ }^{5}$ http://www.ruv.itesm.mx/convenio/tabasco/oas/ureape/homedoc.htm

${ }^{6}$ O OA Incluir cresceu e agora é chamado de Projeto Incluir. Disponível em: 〈https://proincluir.org/> Acesso em: nov./2019
} 
problematização, interação e navegação, o que permite seu uso separadamente ou conforme exigido pelos usuários. Todos os módulos cumprem a mesma proposta e organização pedagógica, estando estruturados em três níveis. Para validar o OA os autores realizaram uma pesquisa por meio da aplicação de 163 questionários sobre seus aspectos técnicos e pedagógicos. O estudo concluiu que o OA é um recurso complementar válido para a formação de professores para inclusão.

Em seu estudo, Esteban (2018) investiga o impacto que o vídeo como OA pode ter no desenvolvimento da competência digital dos professores, a fim de avaliar se esse recurso, quando ensinado a partir de uma taxonomia, poderia ser benéfico para o desenvolvimento de competências-chave específicas ao ensino superior. A investigação contou com um grupo de controle, o qual poderia desenvolver sua competência digital com meios tradicionais (tarefa de ASL - Aquisição de Segunda Língua- e livros didáticos) e o grupo experimental, que poderia melhorar sua competência digital usando o livro didático e realizando uma tarefa de ASL em vídeo. Os resultados mostraram como o vídeo pode ser considerado um OA apropriado que ajuda os participantes a melhorar sua proficiência na língua estrangeira e a desenvolver conhecimentos e habilidades de conteúdo (histórico). E, apesar de existirem evidências de que o vídeo pode oferecer auxílio para o desenvolvimento do DigComp ${ }^{7}$, a falta de melhorias significativas em certos indicadores sugere a ideia de que o vídeo não representa uma ferramenta essencial para tal desenvolvimento, por si só, mas teria que ser complementado por um conjunto de padrões fundamentais e uma combinação de OA.

A partir desse MSL foram encontrados cinco (05) trabalhos, sendo quatro (04) ODEA desenvolvidos para a formação de professores (Q1), que tem como propósito: formar professores de estágio (PETRARCA, 2013); formar professores e educadores para o desenvolvimento sustentável (LUPPI, 2013); realizar formação docente orientada ao desenvolvimento de competências para usar REA (LÓPEZ; MARTÍNEZ; MONTOYA, 2014); realizar formação de professores sobre inclusão e a ressignificação da prática docente (BISOL; VALENTINI; BRAUN, 2015); e um (01) trabalho que analisa o impacto do uso de vídeo como OA no desenvolvimento da competência digital

\footnotetext{
${ }^{7}$ Disponível em: $<$ https://www.erte.dge.mec.pt/sites/default/files/Recursos/Estudos/digcomp_quadro_europeu_de_referenc ia_para_a_competencia_digital.pdf $>$ Acesso em: jan./2020.
} 
dos professores (ESTEBAN, 2018). No entanto, nenhum dos trabalhos trata de um ODEA voltado para a autoria desses ou direcionado para a formação de professores de LE (Q2, Q3).

A geração de dados da investigação de Petrarca (2013) foi por meio do sistema de rastreamento do ATLT. Acompanhar o percurso dos sujeitos no uso do ODEA é importante para compreender seu processo de desenvolvimento e essenciais para o professor planejar suas próximas ações de ensino, baseado na Zona de Desenvolvimento Proximal de seus estudantes.

Os OA desenvolvidos para o estudo de Luppi (2013) oferecem multimodalidade e hipertextualidade para estimular o aprender do usuário e enriquecer o conteúdo. Esses aspectos são muito importantes para propiciar práticas de multiletramentos tanto em contexto de formação de professores, bem como para estudantes de LE. Outra característica desses OA (LUPPI, 2013), é que oferecem liberdade para o usuário seguir seu caminho, o que é interessante, pois as escolhas devem acontecer conforme a necessidade de cada sujeito, além de estimular a autonomia, o que auxilia o estudante no seu processo de construção de conhecimento.

Luppi (2013) explica que cada OA possui três núcleos de objetivos e conteúdo do tema: a) teórico; b) ético-filosófica e c) dimensão operacional prática, que, conforme a autora, têm o intuito de, ao mesmo tempo, promover conhecimentos, atitudes e comportamentos sustentáveis. Salientando a necessidade de associar a prática à teoria reflexão fundamental para a formação de professores.

No OA proposto por López, Martínez e Montoya (2014) o conteúdo é apresentado em seis tópicos como uma pergunta, e o desenvolvimento do conteúdo de cada um foi escrito de forma independente. Além de auxiliar o sujeito a situar sua navegação pelo ODEA, essas perguntas também podem motivar a participação. Esse estudo ainda oferece contribuições quanto ao extenso processo de validação, realizado com especialistas e professores. Mostrando a importância da avaliação do recurso, quanto ao funcionamento, adequação ao público-alvo, questões pedagógicas, entre outros.

Bisol, Valentini e Braun (2015) propõem um OA não linear, onde cada usuário pode fazer escolhas, com começo livre e não hierárquico. Trata-se de um objeto aberto, pois permite a inserção de novos módulos conforme a necessidade, além desses serem 
independentes. No entanto, ao fazer uma opção, todos os módulos são estruturados em três níveis e, assim, quando o sujeito o acessa, existe um caminho planejado para seguir, apresentando linearidade. No Incluir a página inicial do objeto mostra uma imagem de um neurônio, e na tela do computador a imagem se move e os efeitos visuais simulam sinapses ou trocas de energia. A não linearidade é uma característica muito presente no ciberespaço, nos recursos tecnológicos, na hipertextualidade, na multimodalidade e na conectividade que ele oferece. Dessa maneira, construir um ODEA não linear também é possibilitar multiletramentos, autonomia, problematização e, assim, a construção de novos conhecimentos.

Os resultados dos diferentes estudos aprovaram o uso de ODEA para a formação de professores (Q4). Petrarca (2013) destaca como os resultados mostram que há potencial para o desenvolvimento profissional por meio de uma ferramenta baseada na Web como o ALT. Luppi (2013) concluiu que os OA podem ser utilizados em diversos contextos, mas o próprio contexto de uso é uma peça importante para o alcance de resultados satisfatórios. Afinal, outros fatores estão relacionados, os sujeitos possuem um certo desenvolvimento real, assim como estão situados em um contexto cultural e histórico. Por isso, o professor destaca-se como o sujeito mais indicado para construir ODEA para seus estudantes, é o que tem a possibilidade de conhecer maior número de fatores que podem influenciar os processos de ensino e aprendizagem. Os resultados de López, Martínez e Montoya (2014) mostram como o OA possui relevância no desenvolvimento de competências. Destacam como não podemos ignorar o fato de que os avanços tecnológicos estão em ritmo acelerado e, se pretendemos, como educadores, influenciar essa realidade em mudança, é necessário desenvolver competências, superar a resistência e criar OA pertinentes e abertos para toda a comunidade acadêmica.

Bisol, Valentini e Braun (2015) identificaram exemplos de movimentos de construção e reconstrução do conhecimento. Logo, avaliam o OA como recurso complementar valioso, o qual contribui para a formação de professores. Esteban (2018) mostra que os vídeos podem oferecer auxilio no desenvolvimento de competência digitais dos professores, no entanto, uma combinação de OA seria mais indicada.

Para além do exposto, considera-se, ainda, como contribuição as atividades de autoavaliação dos OA de Luppi (2013), as quais possuem o objetivo de acompanhar e oferecer motivação para a aprendizagem, o que é benéfico para situações a distância, 
mas também se apresenta como uma alternativa para realizar a autoavaliação em outras modalidades. Os resultados do estudo apontaram que, além das atividades serem aprovadas pelos participantes, também são interessantes para a motivação em aprender.

\section{Considerações Finais}

O presente Mapeamento Sistemático da Literatura ocupou-se de mapear os Objetos Digitais de Ensino e Aprendizagem (ODEA) existentes para a formação de professores. Em relação às perguntas norteadoras do estudo, a análise possibilitou encontrar quatro (04) ODEA desenvolvidos para a formação de professores (Q1), no entanto, nenhum aborda a questão da formação para a autoria de ODEA ou é direcionado para a formação de professores de LE (Q2; Q3). E todos aprovaram o uso de ODEA para a formação de professores (Q4).

Nesse sentido, fica evidente a potencialidade da utilização de ODEA na formação de professores e, também a necessidade de proporcionar tais momentos para que a TCD possa cada vez mais auxiliar na mediação dos processos de ensino e aprendizagem. Para que dessa forma os ODEA, que se configuram como outras, novas possibilidades de mediar os processos de ensino e aprendizagem de LE, possam ser utilizados cada vez mais nesses contextos.

Ao analisar os cinco (05) trabalhos selecionados, possibilidades e caminhos para a formação de professores por meio de ODEA foram encontrados. No entanto, as reflexões não se esgotam. Os resultados desta investigação identificam uma coletânea de pesquisas na área, e, dessa forma, podem oferecer a pesquisadores um panorama sobre o uso de ODEA para a formação de professores e sobre processos de mediação por meio de tecnologias. Como trabalho futuro, pretende-se oferecer formação sobre ODEA para professores de Língua Estrangeira com o intuito de proporcionar referências que possam embasar suas práticas.

\section{REFERÊNCIAS}

COSTA BARCELloS, Patrícia Silva Campelo. O Processo de Criação Colaborativa de Tarefas em Língua Estrangeira em Ambiente digital por Professores em Formação. 2014. Tese (Doutorado em Linguística Aplicada) - Universidade do Vale do Rio dos Sinos. São Leopoldo, RS, 2014. 
BISOL, Cláudia Alquati; VALENTINI, Carla Beatris; BRAUN, Karen Cristina Rech. Teacher education for inclusion: Can a virtual learning object help? Computers \& Education, v. 85, p. 203-210, 2015.

DERMEVAL, Diego; COELHO, Jorge A. P. de M.; BITTENCOURT, Ig I. Mapeamento Sistemático e Revisão Sistemática da Literatura em Informática na Educação. In: JAQUES, Patrícia Augustin; SIQUEIRA; Sean; BITTENCOURT, Ig; PIMENTEL, Mariano. (Org.) Metodologia de Pesquisa Científica em Informática na Educação: Abordagem Quantitativa. Porto Alegre: SBC, 2020. (Série Metodologia de Pesquisa em Informática na Educação, v. 2) Disponível em: <https://metodologia.ceie-br.org/livro-2> Acesso em: jun./2019.

EADY, Michelle; LOCKYER, Lori. Tools for learning: technology and teaching strategies. In: HUDSON, Peter (Ed.) Learning to Teach in the Primary School. Queensland: Queensland University of Technology, 2013, p. 71-89.

ESTEBAN, Soraya García. Do Video Learning Objects Develop Digital competence in Teacher Training? RAEL: Revista Electrónica de Lingüística Aplicada, España, v. 16, n. 1, p. 85-102, 2018.

KITCHENHAM, Barbara; CHARTERS, Stuart. Guidelines for performing Systematic Literature Reviews in Software Engineering. Technical Report EBSE 2007-001, Keele University and Durham University Joint Report, 2007.

LÉVY, Pierre. Cibercultura. São Paulo: ed. 34, 1999.

LÓPEZ, Ahabael Lugo; MARTÍNEZ, Fernando Gustavo Lozano; MONTOYA, María Soledad Ramírez. Objeto de Aprendizaje para la Formación Docente Orientado a Desarrollar Competencias para Usar REA. TESI: Teoría de la Educación: Educación y Cultura en la Sociedad de la Información, España, v. 15, n. 2, p. 32-48, jul., 2014.

LUDOVICO, Francieli Motter; COSTA BARCELLOS, Patrícia da Silva Campelo. Momentos de Reflexão Docente Frente à Sociedade da Informação: inventividade e produção de subjetividades. Tear: Revista de Educação Ciência e Tecnologia, v. 8, n. 2, 2019.

LUPPI, Elena. Progettazione e valutazione di un percorso formativo basato sui Learning Object per chi educa allo sviluppo sostenibile. Ricerche di Pedagogia e Didattica Journal of Theories and Research in Education, Bologna, v. 8, n. 1, p. 21, jun., 2013.

MOHER, David; STEWART, Lesley; SHEKELLE, Paul. All in the Family: systematic reviews, rapid reviews, scoping reviews, realist reviews, and more. Systematic Reviews 4, 183, 2015.

PETRARCA, Diana. A Promising Practicum Pilot - Exploring Associate Teachers' Access and Interactions with a Web-based Learning Tool. Interdisciplinary Journal of E-Learning and Learning Objects, Australia, v. 9, n. 1, p. 149-170, jan., 2013. 
RAMOS, Rosinda de Castro Guerra; RAMOS, Simone Telles Martins; ASEGA, Fernanda Katherine. Google Drive: Potencialidades para o Design de Material Educacional Digital (med) para ensino de línguas. In: The ESPecialist: Descrição, Ensino e Aprendizagem, Vol. 38 No. 1 jan-jul 2017.

ROJO, Roxane. Entre Plataformas, ODAS e Protótipos: Novos Multiletramentos em Tempos de WEB2. The ESPecialist: Descrição, Ensino e Aprendizagem, São Paulo, Vol. 38 No. 1, p. 1-20, jan-jul, 2017.

VYGOTSKY, Lev Semionovich. A formação social da mente: o desenvolvimento dos processos psicológicos superiores. $4^{\mathrm{a}}$ ed, São Paulo: Martins Fontes, 1991.

ZACHARIAS, Valéria Ribeiro de Castro. LETRAMENTO DIGITAL: desafios e possibilidades para o ensino. - In: COSCARELLI, Carla Viana (org). Tecnologias para aprender. São Paulo: Parábola Editorial, 2016.

Recebido em 26/05/2020. Aceito em 15/06/2020. 